\title{
FECHANDO HUESO CREMADO: LA BASE CIENTÍFICA $(1)$
}

\author{
DATING CREMATED BONE: \\ THE SCIENTIFIC BACKGROUND
}

\author{
J.N. LANTING $(*)$ \\ A.L. BRINDLEY $(*)$
}

\section{RESUMEN}

Los autores presentan un nuevo método para obtener fechas de datación absoluta a partir de restos de hueso sometidos a diferentes grados de combustión y explican algunas de sus recientes experiencias y los resultados obtenidos.

\begin{abstract}
A new method of obtaining absolute chronology dates out of burnt or cremated bone is presented. The authors explain their recent experiments and give promising results.
\end{abstract}

Palabras clave: Datación. Hueso quemado. Cronología absoluta. Metodología.

Key words: Dating. Cremated bone. Absolute chronology. Methodology.

El hueso consiste en largas cadenas de proteínas (colágeno) en las que se intercalan partículas de materia inorgánica escasamente cristalizadas. Esta materia inorgánica es en esencia fosfato cálcico con estructura parecida a una apatita (bio-apatita). Una característica de esta bioapatita es que incorpora determinada cantidad de carbonato $(0,5-1 \%)$ susti-

(1) El original en inglés fue traducido por Alicia Perea (Dpto. de Prehistoria. Instituto de Historia. CSIC. Serrano 13. 28001 Madrid) y revisado por F. Alonso Mathias (Instituto de Química Física Rocasolano, Laboratorio de Geocronología. Serrano 119, 28006 Madrid).

(*) Jan N. Lanting y Anna L. Brindley. Dept. of Archaeology. Rijksuniversiteit. Poststraat, 6. 9712 ER. Groningen. The Netherlands.

El artículo fue remitido en su versión final el 2-X-1999.

tuyendo al fosfato en la red cristalina. El llamado carbonato estructural tiene su origen en el bicarbonato de la sangre que se genera en las células al producir energía; por tanto, está en relación directa con la comida ingerida por la persona o el animal en cuestión. El carbonato estructural es de gran interés para los estudios sobre paleo-dieta; consecuentemente, se han desarrollado y ensayado métodos para obtener carbonato estructural a partir de la bioapatita y separarlo del carbonato "absorbido" por los huesos arqueológicos (Lee-Thorp et alii, 1989; Lee-Thorp y Van der Merwe, 1991; Ambrose y Norr, 1993). También se ha utilizado para datación radiocarbónica, aunque en menor medida. El carbonato procedente del esmalte dental no quemado (muy parecido a la bioapatita desde el punto de vista químico) proporcionaba fechas aberrantes supuestamente debidas a cambios post-deposicionales (Hedges et alii, 1995).

En el Tercer Simposio Internacional de $14 \mathrm{C}$ y Arqueología del 6 al 10 de Abril de 1998 en Lyon, un grupo de científicos franceses (Saliège et alii, 1998; Person et alii, 1998) presentaron los resultados de un programa de datación basado en el carbonato estructural de esqueletos prehistóricos del Sahel. Estas fechas de carbonato pudieron contrastarse con fechas de colágeno, carbón vegetal o hueso quemado, resultando ser fiables. Debido al clima extremadamente seco de la zona, no existían cambios post-deposicionales. Damos los resultados en la tabla 1.

Después de haber escuchado esta comunicación de Lyon, uno de nosotros (JNL) se dió cuenta que sería posible fechar hueso cremado debido a los cambios que ocurren durante el proceso de crema- 


\begin{tabular}{|l|l|l|l|}
\hline \multicolumn{1}{|c|}{ Yacimiento } & Ref. & \multicolumn{1}{c|}{ Edad BP } & \multicolumn{1}{c|}{ Edad BP } \\
\hline Mali Tioubel & $\mathrm{Pa}-788$ & $1560 \pm 80$ & $1600 \pm 80$ \\
\hline Algerie Torba & $\mathrm{Pa}-1257$ & $1780 \pm 60$ & $1790 \pm 60$ \\
\hline Mali Kobadi & $\mathrm{Pa}-1341$ & $3450 \pm 80$ & $3335 \pm 100$ \\
\hline Chin Tafidet & $\mathrm{Pa}-1054$ & $3910 \pm 80$ & $3830 \pm 75$ \\
\hline In Tuduf & $\mathrm{Pa}-1062$ & $3540 \pm 70$ & $3415 \pm 100$ \\
\hline Afunfun & $\mathrm{Pa}-1039$ & $3760 \pm 80$ & $3850 \pm 100$ \\
\hline Algerie T'Hanakaten & $\mathrm{Pa}-1083$ & $8230 \pm 140$ & $8410 \pm 60$ \\
\hline
\end{tabular}

Tab. 1. Resultados del equipo francés sobre carbonato de hueso no quemado versus colágeno/carbón/hueso quemado del mismo contexto (columna derecha).

ción. Con anterioridad, todos los intentos de fechar hueso cremado habían fracasado porque los laboratorios de radiocarbono trataban el hueso cremado del mismo modo que el hueso quemado. El hueso quemado ha sido sometido a temperaturas relativamente bajas $\left(200-300^{\circ} \mathrm{C}\right)$, contiene grasas y proteínas carbonizadas, y tiene el interior gris o negro. El hueso cremado ha sido sometido a una temperatura bastante más alta (por encima de los $600^{\circ} \mathrm{C}$ ), no contiene en absoluto materia carbonizada, y es blanco por todos lados. Durante un proceso de incineración a temperatura superior a los $600^{\circ} \mathrm{C}$, la bioapatita recristaliza formando cristales más grandes y con mejor estructura (Shipman et alii, 1984). Esta es una de las razones por las que el hueso cremado se conserva, aún en suelo ácido. Al mismo tiempo, sin embargo, desaparece cierta cantidad del carbonato estructural (Stiner et alii, 1995).

Al considerar muy poco probable que todo el carbonato estructural desaparezca en una pira prehistórica, pedimos al laboratorio de Groningen que datase el carbonato estructural de determinado número de cremaciones. Éstas habían sido datadas previamente utilizando el carbón encontrado entre los huesos cremados, de forma que fue posible contrastar sus resultados con los del carbonato.

Resultó que las cremaciones efectivamente contenían suficiente carbonato estructural para hacer una datación porAMS, aunque en algunos casos no superase el $0,1 \%$. La proporción estable del isótopo d13C mostraba que una cantidad considerable de carbonato debió desaparecer quemada, teniendo como resultado un cambio notable en el d13C, debido al fraccionamiento isotópico durante el proceso. Sin embargo, esto no condicionaba la databilidad del hueso cremado. Los resultados de los ensayos aparecen en la tabla 2. Demuestran que no se requiere más de 1,5-2 $\mathrm{g}$ de hueso cremado para

\begin{tabular}{|c|c|c|c|c|}
\hline Yacimiento & & Ref. & Edad & Edad \\
\hline Anlo-Molenes & & GrA-11256 & $2970 \pm 40$ & $2945 \pm 35$ \\
\hline Hijkeres & & GrA-11259 & $1760 \pm 50$ & $1720 \pm 30$ \\
\hline Oudemolen & & GrA-11263 & $2460 \pm 50$ & $2345 \pm 35$ \\
\hline Rolde & & GrA-11264 & $3840 \pm 50$ & $3850 *$ \\
\hline Vledder 271 & & GrA-11666 & $2750 \pm 40$ & $2800 *$ \\
\hline Vledder 296f & & GrA-11667 & $2930 \pm 40$ & $2900 *$ \\
\hline \multirow[t]{2}{*}{ Eext 1952} & $\mathrm{~S}$ & GrA-11675 & $2760 \pm 40$ & $2785 \pm 35$ \\
\hline & $\mathrm{F}$ & Gra-18876 & $2670 \pm 50$ & \\
\hline \multirow[t]{2}{*}{ Wapse 58} & $\mathrm{~S}$ & GrA-11669 & $2540 \pm 40$ & $2580 \pm 40$ \\
\hline & $\mathrm{F}$ & GrA-11671 & $2530 \pm 40$ & \\
\hline \multirow[t]{2}{*}{ Wapse 130} & $\mathrm{~S}$ & GrA-11672 & $2580 \pm 40$ & $2390 \pm 35$ \\
\hline & $\mathrm{F}$ & GrA-11674 & $2510 \pm 40$ & \\
\hline \multirow[t]{2}{*}{ Eext 1967} & $\mathrm{~S}$ & GrA-11676 & $2230 \pm 40$ & $2345 \pm 35$ \\
\hline & $\mathrm{F}$ & GrA-11677 & $2210 \pm 40$ & \\
\hline \multirow[t]{2}{*}{ Gasteren 1939} & $\mathrm{~S}$ & GrA-10877 & $2910 \pm 50$ & $2900 *$ \\
\hline & $\mathrm{F}$ & GrA-10880 & $2860 \pm 60$ & \\
\hline $\begin{array}{l}\text { Hoogeloon-Broeken- } \\
\text { eind viii }\end{array}$ & & GrA-13367 & $1490 \pm 40$ & c. $1450 *$ \\
\hline $\begin{array}{l}\text { Hoogeloon-Broeken- } \\
\text { eind ii }\end{array}$ & & GrA-13368 & $1530 \pm 30$ & c. $1500 *$ \\
\hline Wijster graf xxiv & & GrA-13369 & $1600 \pm 40$ & $1600-1150 *$ \\
\hline Wijster graf xix & & GrA-13370 & $1700 \pm 40$ & $1700-1650 *$ \\
\hline Besthmen 1937/iii.1 & & GrA-13372 & $1780 \pm 40$ & $1800-1700^{*}$ \\
\hline $\begin{array}{l}\text { Havelterberg 1943/ } \\
\text { iii.1 }\end{array}$ & & GrA-13374 & $2120 \pm 40$ & c. $2100 *$ \\
\hline $\begin{array}{l}\text { Laudermarke 1998- } \\
3-2\end{array}$ & & GrA-13375 & $2220 \pm 40$ & $2290 \pm 30$ \\
\hline Oirschot V-21 & & GrA-13390 & $8320 \pm 40$ & $7790 \pm 130$ \\
\hline Kettig & & GrA-13389 & $11.710 \pm 50$ & $11.314 \pm 50$ \\
\hline $\begin{array}{l}\text { Doetinchem-Dich- } \\
\text { teren } 8\end{array}$ & & GrA-13387 & $10.880 \pm 50$ & $10.870 \pm 50$ \\
\hline $\begin{array}{l}\text { Doetinchem-Dich- } \\
\text { teren } 17\end{array}$ & & GrA-13388 & $10.930 \pm 50$ & $10.870 \pm 50$ \\
\hline
\end{tabular}

Tab. 2. Resultados del laboratorio de Groningen (CIO) sobre carbonato de hueso cremado versus carbón asociado o edad estimada $(*)$ basada en fechas de carbón de hallazgos y/o estructuras relacionadas (columna derecha). En algunos casos se han fechado tanto fragmentos $(\mathrm{F})$ como huesos compactos (S) de la misma cremación. La muestra de carbón de Oirschot V-21 fue tratada sólo con ácido previamente y la edad es claramente demasiado baja.

una datación, y que se pueden utilizar pequeños fragmentos, incluidos los huesos porosos, en vez de fragmentos más grandes de hueso compacto. De especial interés son las fechas de un conjunto de huesos humanos, en parte chamuscados y en parte cremados, de un nivel de turbas margosas cerca de Diepenveen (ver tabla 3).

Podemos asumir que la "vida" del carbonato estructural es corta, y del mismo orden de magnitud que la "vida" del colágeno del hueso, pongamos unos 15-20 años como mucho. La bioapatita y el

T. P., 56, n. ${ }^{\circ} 2,1999$ 


\begin{tabular}{|c|l|c|}
\hline Ref. & \multicolumn{1}{|c|}{ Material } & Edad \\
\hline GrN-24172 & hueso chamuscado (carbón) & $2420 \pm 100$ \\
\hline GrA-11680 & hueso cremado (carbonato) & $2480 \pm 40$ \\
\hline GrN-24291 & turba & $2520 \pm 80$ \\
\hline
\end{tabular}

Tab. 3. Fechas de varias muestras de hueso humano, en parte quemado y en parte cremado, procedente de una turbera cerca de Diepenveen, Holanda.

colágeno de los huesos son reemplazados en vida a un ritmo lento pero constante. Debemos hacer hincapié, por tanto, en que fechar el carbonato estructural en la bioapatita no tiene nada que ver con fechar el "carbonato del hueso" según se hacía en los primeros años de las dataciones de radiocarbono. De hecho, el "carbonato del hueso" era el carbonato absorbido por la superficie de los cristales de bioapatita. Su origen estaba en el dióxido de carbono o carbonato del suelo, procedente de fuentes diversas, que rodeaba al hueso en cuestión. No sorprendía que estas fechas de carbonato "absorbido" fueran aberrantes en la mayoría de los casos y que, por tanto, desprestigiasen la datación radiocarbónica del hueso hasta que se normalizó la datación del colágeno. Cuando fechamos carbonato estructural, el carbonato "absorbido" se elimina con la preparación de la muestra.

Despues de obtener estos prometedores resultados para el hueso cremado, decidimos ensayar las posibilidades de datación del hueso no quemado, a pesar de los resultados negativos obtenidos previamente por el programa sobre esmalte dental de Oxford. Se eligieron siete muestras ya fechadas por colágeno. Para nuestra sorpresa, no aparecieron diferencias significativas entre las fechas del carbonato y las del colágeno (Tab. 4).

Todavía no es totalmente seguro que el carbonato del hueso no quemado sea siempre un buen sustituto del colágeno. Las fechas del colágeno y del

\begin{tabular}{|l|c|c|c|}
\hline \multicolumn{1}{|c|}{ Yacimiento } & Ref. & Edad & Edad \\
\hline Dillonsdown & GrA-13330 & $3730 \pm 50$ & $3660 \pm 70$ \\
\hline Grange 2 & GrA-13331 & $3600 \pm 40$ & $3620 \pm 80$ \\
\hline Grange 3 & GrA-13332 & $3740 \pm 40$ & $3770 \pm 70$ \\
\hline Knowth 7 & GrA-13334 & $1880 \pm 40$ & $1920 \pm 60$ \\
\hline Knowth 14 & GrA-13335 & $1260 \pm 40$ & $1270 \pm 25$ \\
\hline Phoenix Park & GrA-10970 & $4520 \pm 80$ & $4650 \pm 70$ \\
\hline Topped Mtn & GrA-13333 & $3520 \pm 40$ & $3460 \pm 50$ \\
\hline
\end{tabular}

Tab. 4. Resultados de CIO sobre carbonato en hueso no quemado, comparados con fechas de colágeno del mismo hueso (columna derecha). carbonato de un colmillo de mamut procedente de Rusia difieren considerablemente: $17.720 \pm 120$ (GrA-11812) frente a 9.930 $\pm 50 \mathrm{BP}(\mathrm{GrA}-11679)$. En este caso, sin embargo, no está claro cuál de las dos es la correcta. Es necesario, por tanto, más trabajo sobre hueso no quemado.

Desde un punto de vista teórico, el carbonato estructural tiene claras ventajas sobre el colágeno. Éste tiene su origen en las proteínas de los alimentos, y es propenso al efecto "pescado" cuando la dieta contiene cantidades considerables de pescado de agua salada o dulce y marisco. En estos casos las fechas de colágeno serán demasiado altas (Lanting y Van der Plicht, 1995-96). El carbonato estructural tiene su origen en la dieta completa, por tanto, también en los carbohidratos y grasas, que en una dieta "normal" son las fuentes principales de energía. Consecuentemente el carbonato estructural producirá fechas más fiables (2).

\section{BIBLIOGRAFÍA}

Ambrose, S.E. y Norr, L. (1993): "Experimental evidence for the relationship of the carbon isotope ratios of whole diet and dietary protein to those of bone collagen and carbonate". En J.B. Lambert y Grupe G. (eds.): Prehistoric human bone. Archaeology at the molecular level. Springer Verlag. Berlin: 1-37.

Hedges, R.E.M.; Thorp, J.A. y Tuross, N.C. (1995): "Is tooth-enamel carbonate a suitable material for radiocarbon dating?". Radiocarbon, 37: 285-290.

Lanting, J.N. y Plicht, J. van der (1995-96): "What do Count Florence V, skeleton Swifterbant S2 and others have in common?". Palaeohistoria, 37/38: 512-516.

LEE-Thorp, J.A. y Merwe, N.J. van der (1991): "Aspects of the chemistry of modern and fossil biological apatites". Journal of Archaeological Science, 18: 43-354.

Lee-Thorp, J.A.; Sealey, J.C. y Merwe, N.J. van der (1989): "Stable carbon isotope ratio differences between bone collagen and bone apatite, and their relationship to diet". Journal of Archaeological Science, 16: 585-599.

Person, A.; Saliège, J.-F.; Gérard, M. y Paris, F. (1998): "Utilisation d'un indice caractéristique de la diagenèse de la fraction minéral d'ossements archéologiques en milieu desértique pour discuter de la fiabilité de ces matériaux comme support de datation par le radiocar-

(2) La datación del carbonato estructural en huesos cremados está comercialmente disponible en el laboratorio de radiocarbono de Groningen. La persona de contacto: Dr. J. Van der Plicht en el Centro de Investigación de Isótopos, Nijenborgh 4, 9747 AG Groningen, Holanda. Tel.: (50) 3634760, Fax: (50) 3634738 
bone, application à deux necropoles neolithique de l'Aür (Niger)". Pré-actes du 3ème Congrés international ${ }^{14} \mathrm{C}$ et archéologie, Lyon 1998: 77-78.

Saliège, J.-F.; Person, A. y PARIS, F. (1998): "Datation du carbonate-hydroxylapatite d'ossements holocenes du Sahel (Mali, Mauritanie, Niger)". Pré-actes du 3ème Congrés international ${ }^{14} \mathrm{C}$ et archéologie, Lyon 1998: 172-173.
Shipman, P.; Foster, G.F. y Schoeninger, M. (1984): "Burnt bones and teeth: an experimental study of colour, morphology, crystal structure and shrinkage". Journal of Archaeological Science, 11: 307-325.

Stiner, M.C.; Kuhn, S.L.; Weiner, S. y Bar-Yosef, O. (1995): "Differential burning, recrystallization, and fragmentation of archaeological bone". Journal of Archaeological Science, 22: 223-237. 